Website: http://revistas.lamolina.edu.pe/index.php/acu/index

(C) Universidad Nacional Agraria La Molina, Lima - Perú

\title{
Evaluación del fotoperiodo en el asentamiento de tetraesporas de Chondracanthus chamissoi sobre cuerdas de polipropileno en condiciones semi-controladas de laboratorio
}

\author{
Evaluation of the photoperiod in the settlement of Chondracanthus chamissoi tetraspores on \\ polypropylene strings in semi-controlled laboratory conditions
}

\author{
Max Castañeda ${ }^{1,2}$, Samuel Arbaiza ${ }^{1,2}$, Francisco Diaz $^{1}$, Yorka Castillo ${ }^{1}$, Paul Baltazar $^{3}$, Orlando Advíncula ${ }^{4}$ \\ * Autor de correspondencia
}

\section{Resumen}

Las macroalgas rigen su carácter reproductivo con relación al fotoperiodo y la temperatura del medio ambiente en donde se desarrollan. El género Chondracanthus presenta un ciclo de vida trifásico isomórfico y su reproducción es por esporas. A fin de evaluar las mejores condiciones en que se produce liberación de tetrasporas, una biomasa total de 120 gramos de la fase tetrasporofitica de Chondracanthus chamissoi fue sometida a estrés durante tres horas de desecación y luego colocado en matraces con agua de mar enriquecida para inducir la liberación de esporas. Posteriormente estas esporas fueron colocadas en envases con sustrato artificial a fin de conocer el número de esporas asentadas por centímetro de cuerda (nEA), diámetro promedio del disco de germinación ( $\mathrm{dDG}$ ) y porcentaje promedio de discos con formación de microtalo (\%dM). Se evaluo tres tratamiento de fotoperiodo 16:08, 12:12 y 08:16 (L:O). Se encontró que existen diferencias significativas $(\mathrm{p}<0,05)$ en la cantidad de tetrasporas liberadas por cada tratamiento de fotoperiodo (T1: 0,98*104 esp/ml; T2: 1,65*104 esp/ml; T3: 2,54*104 esp/ml). A nivel de cultivo, los valores de nEA (T1: 25,3 $\pm 0,3$; T2: 26,0 $\pm 0,3 ; \mathrm{T} 3: 27,3 \pm 0,3)$ y dDG (T1: $750 \pm 35 \mu \mathrm{m}$; T2: $730 \pm 20 \mu \mathrm{m}$; T3: $775 \pm 20 \mu \mathrm{m})$ no presentaron diferencias significativas $(\mathrm{p}>0,05)$ en todos los sistemas trabajados, sin embargo si se presentaron diferencias significativas $(\mathrm{p}<0,05)$ en los valores \%dM (T1: 79,5 $\pm 5,5 \%$; T2: 85,5 $\pm 3,5 \%$; T3: 100,5 \pm 0,5 \%). El trabajo realizado indica que el fotoperiodo 08:16 (L:O) tiene un mejor efecto en el asentamiento y crecimiento de tetrasporas de Chondracanthus chamissoi.

Palabras clave: Chondracanthus chamissoi; yuyo; chicorea de mar; tetrasporas; cultivo algas; fotoperiodo.

\begin{abstract}
Macroalgae govern their reproductive character in relation to the photoperiod and the temperature of the environment in which they develop. The genus Chondracanthus presents an isomorphic three-phase life cycle and its reproduction is by spores. In order to evaluate the best conditions in which tetraspore release occurs, a total biomass of 120 grams of the tetrasporophytic phase of Chondracanthus chamissoi was stressed for three hours of desiccation and then placed in flasks with enriched seawater to induce release of spores. Later these spores were placed in containers with artificial substrate in order to know the number of spores seated per centimeter of rope (nEA), average diameter of the germination disk (dDG) and average percentage of discs with microtalus formation $(\% \mathrm{dM})$. We evaluated three photoperiod treatment 16:08, 12:12 and 08:16 (L: $\mathrm{O})$. It was found that there are significant differences $(\mathrm{p}<0.05)$ in the amount of tetraspores released by each photoperiod treatment (T1: $0.98 * 104 \mathrm{sp} / \mathrm{ml}, \mathrm{T} 2: 1,65 * 104 \mathrm{sp} / \mathrm{ml}, \mathrm{T} 3: 2,54 * 104 \mathrm{sp} / \mathrm{ml})$. At the culture level, the NEA values (T1: 25,3 $\pm 0,3, \mathrm{~T} 2: 26,0 \pm 0,3 ; \mathrm{T} 3: 27,3 \pm 0,3)$ and $\mathrm{dDG}(\mathrm{T} 1: 750 \pm 35 \mu \mathrm{m}, \mathrm{T} 2: 730 \pm 20 \mu \mathrm{m}, \mathrm{T} 3: 775$ $\pm 20 \mu \mathrm{m})$ did not present significant differences $(\mathrm{p}>0,05)$ in all systems worked, however if significant differences were found $(\mathrm{p}<0,05)$ in the $\% \mathrm{dM}$ values $(\mathrm{T} 1: 79,5 \pm 5,5 \%, \mathrm{~T} 2: 85,5 \pm 3,5 \%, \mathrm{~T} 3: 100,5 \pm 0,5 \%)$. The work done indicates that the photoperiod 08:16 (L: O) has a better effect on the settlement and growth of tetraspores of Chondracanthus chamissoi.
\end{abstract}

Keywords: Chondracanthus chamissoi; yuyo; chicorea de mar; tetraspores; algae culture; photoperiod.

\section{Introducción}

Chondracanthus chamissoi (C. Agardh) Kützing comúnmente conocida como "yuyo", "mococho" o "chicorea de mar", es un alga roja endémica de la costa del Pacífico Sudamericano (5 - $\left.42^{\circ} \mathrm{S}\right)$ (Dawson et al., 1964; Ramírez \& Santelices, 1991), que ha sido comúnmente objeto de extracción debido a su importancia comercial como fuente de carragenano y para consumo humano directo (Acleto, 1971; Bulboa et al., 2013). Es debido a esa importancia que diversas investigaciones relacionadas al conocimiento del cultivo de $C$. chamissoi han sido llevadas a cabo, principalmente en el país de Chile, tales como el desarrollo de cultivos vegetativos por medio de talos fragmentados (Bulboa et al., 2005; Bulboa 2006; Bulboa \& Macchiavello, 2006; Bulboa et al., 2013) y sobresalientemente en el cultivo vía esporas (Bulboa \& Macchiavello, 2001; Bulboa et al., 2010).

Para el Perú el cultivo vegetativo ha sido ampliamente

\footnotetext{
${ }^{1}$ Acuícola Mares del Sur. Lima, Perú. E-mail: maxbiomar23@gmail.com

${ }^{2}$ Universidad Nacional Agraria La Molina. Laboratorio de Biología Aplicada, Lima, Perú.

${ }^{3}$ Universidad Científica del Sur, Lima, Perú.

${ }^{4}$ Facultad de Ingeniería. Universidad San Ignacio de Loyola. Lima, Perú.
} 
desarrollado y estudiado, sin embargo, el cultivo vía esporas o esporocultivo es una tecnología recientemente aplicada y puesta en marcha que requiere aun gran investigación a fin de obtener datos relevantes sobre la capacidad reproductiva que tienen las diversas praderas de C. chamissoi. Al igual que las demás Gigartinales, el comportamiento reproductivo de $C$. chamissoi en cuanto a la abundancia de carposporas y tetraesporas en cualquier época del año es función de: (1) la abundancia relativa de la fase reproductiva correspondiente, (2) el número de estructuras por fronda y (3) número de esporas por estructura reproductiva (Gonzálezy Meneses, 1996; Bulboa et al., 2010). González y Meneses (1996) informaron para el norte de Chile (Puerto Aldea, $29^{\circ}-30^{\circ} \mathrm{S}$ ), que durante el verano era mayor la producción de esporas, la germinación y el reclutamiento de nuevas plántulas enfocadas en la fase carpospórica; mientras que Vásquez y Vega (2001) informaron que estas características se dieron en primavera. Sin embargo, en Perú las praderas naturales de esta especie muestran una relación estacional para la capacidad de producción de esporas por estructura reproductiva para las fases tetrasporofitica y carposporofitica observado en invierno y primavera respectivamente.

\section{Caracterización de la especie}

C. chamissoi habita en las zonas rocosas del intermareal y submareal creciendo tanto en regiones expuestas al oleaje como protegidas hasta los 15 metros de profundidad (Bulboa \& Macchiavello, 2006, Calderón et al., 2010). Tiene un talo membranoso y cartilaginoso (Calderón et al., 2010). Esta especie es morfológicamente muy variable (Acleto, 1986) con una gran variabilidad de colores.

C. chamissoi tiene un ciclo de vida trifásico e isomórfico con alternancia de esporofitos y gametofitos erectos (Calderón et al., 2010; Ávila et al., 2011). La fase sexual o gametofítica haploide, está representada por los gametofitos masculinos y femeninos (gametofitos dioicos). Los gametofitos masculinos producen los espermacios (gameto masculino) que son arrastrados por la corriente hasta el carpogonio (gameto femenino) (Macaya, 2001). Después de la fecundación, los cistocarpos que se desarrollan sobre el gametofito femenino, que representan la fase cistocárpica o carposporofito diploide, forman carposporas (diploides) que germinan dando lugar a la fase asexual libre o tetrasporofito diploide, de morfología similar a los gametofitos (Acleto, 1986). Los individuos tetrasporofíticos poseen pequeños soros tetrasporangiales donde se producen tetrasporas (esporas haploides). Luego de ser liberadas, las tetrasporas germinan originando gametofitos haploides femeninos o masculinos. Los gametofitos femeninos (cistocárpicos), gametofitos masculinos y tetrasporofitos son morfológicamente semejantes, sin embargo, al mismo tiempo son fáciles de ser diferenciados, aun mostrando tamaño y coloración diferente (Bulboa \& Macchiavello, 2006).

C. chamissoi tiene estrategias reproductivas que permiten su mantenimiento en praderas naturales tales como la producción de gametos, la producción de carposporas y tetrasporas, la fragmentación del talo (Bulboa et al., 2013) y la formación de un sistema basal crustoso permanente sobre el sustrato que tiene la capacidad de regenerar frondas (Alveal, 2001).

Además determinaron que las esporas tienen una marcada estacionalidad las cuales se producen principalmente en primavera y verano presentando una marcada sincronización entre la fertilidad y el período de mayor crecimiento vegetativo (Bulboa et al., 2010).

\section{$\underline{\text { Factores que afectan su cultivo }}$}

Estos factores han sido determinados por diversos estudios, siendo los principales la temperatura, fotoperiodo, irradiación y la disponibilidad de nutrientes (Lobban \& Harrison, 1994; Oliveira et al., 1995; Edding, 1995; Alveal et al., 1995; Bulboa \& Macchiavello, 2001; Riofrío, 2003; Bulboa, 2006, Barsanti \& Gualtieri, 2014).

La temperatura afecta principalmente las tasas de reacciones químicas y el metabolismo correlacionándose directamente con la fotosíntesis, la respiración, la inducción a la esporulación, germinación, el crecimiento y desarrollo de las algas (Lobban \& Harrison, 1994). Diversos investigadores han detallado la importancia de la temperatura en el cultivo de algas (Salinas \& Valdés, 1993; Oliveira et al., 1995; Edding, 1995; Garza-Sánchez et al., 2000; Macaya, 2001; Agrawal, 2001; Bulboa \& Macchiavello, 2001; Buschmann et al., 2004; Bulboa, 2006; Redmond et al., 2014). Por lo tanto, es importante en un cultivo tratar de mantener las condiciones de temperatura en condiciones óptimas para el crecimiento de la especie; esto puede ser por medio de sistemas de refrigeración, calentamiento y circulación de aire según sean las particularidades del alga cultivada (Oliveira et al., 1995).

Se sabe que en los organismos fotoautótrofos, como C. chamissoi, la ganancia neta de biomasa está esencialmente vinculada con el proceso de fotosíntesis (Israel, 1995), ya que incorporarán material inorgánico en biomasa orgánica utilizando la energía del sol (Redmond et al., 2014). Esta energía del sol está caracterizada por la cantidad (fotoperiodo) y calidad (intensidad) de la luz. El fotoperiodo es la cantidad de horas luz y oscuridad del medio natural en la que crece y se desarrolla el alga. Así mismo, la intensidad lumínica, flujo fotónico o irradiación es la medida de la cantidad de energía que cae sobre una superficie plana (Lobban \& Harrison, 1994) y está implicada en la alteración de la tasa de división celular interfiriendo en el crecimiento (Bulboa, 2006). Diversos autores han determinado el efecto del fotoperiodo y la irradiación en el crecimiento de algas de un cultivo (Salinas \& Valdés, 1993; Garza-Sánchez et al., 2000; Bulboa \& Macchiavello, 2001; Buschmann et al., 2004; Bulboa, 2006; Ávila et al., 2011; Redmond et al., 2014).

Los nutrientes, los cuales son el conjunto de elementos químicos inorgánicos (incluido las vitaminas), cumplen roles esenciales en el metabolismo de las algas. Tenemos los 
macronutrientes ( $\mathrm{C}, \mathrm{N}$ y P) y micronutrientes o elementos traza (Fe, $\mathrm{Cu}, \mathrm{Mn}, \mathrm{Zn}$, etc.) los cuales son incorporados en los tejidos, estructuras celulares, enzimas, entre otros. Diversos trabajos han sugerido el uso de fertilizantes agrícolas como alternativa de bajo costo al uso de sales inorgánicas de grado analítico para el cultivo masivo (Edding, 1995; Pacheco - Ruíz et al., 2004; Bulboa, 2006; Bulboa et al., 2013). Finalmente, otras variables que afectan el crecimiento y desarrollo de las algas en un cultivo es la turbulencia ocasionada por el movimiento del agua, el pH y la salinidad.

\section{Cultivo de C. chamissoi a partir de esporas}

El cultivo a partir de esporas o esporocultivo consiste en permitir que las esporas (tetrasporas o carposporas) sean inoculadas sobre sustratos artificiales (cuerdas, redes, etc.) para posteriormente ser trasladado a las áreas de cultivo en el mar para su crecimiento y su posterior cosecha (Macaya, 2001). Este sistema de cultivo tiene la ventaja de utilizar una baja cantidad de biomasa reproductiva para iniciar el cultivo (Macaya, 2001; Bulboa et al., 2013) permitiendo un cultivo masivo a partir de cantidades pequeñas de material fértil, abriendo la posibilidad de seleccionar cepas para la producción de plantas de mejor calidad (Alveal et al., 1995). Además, se puede controlar el crecimiento de plantas tetrasporofíticas, gametofíticas o una combinación de ambas, lo que permite cultivar determinada fase en función a los requerimientos del mercado (Macaya, 2001). Adicionalmente, el cultivo a partir de esporas genera una importante variabilidad genética en los cultivos. Sin embargo, diversas experiencias observadas en el medio natural y en procesos de cultivo han determinado la existencia de una alta tasa de mortalidad en las primeras etapas de desarrollo (asentamiento y germinación) (Santelices, 1990; Glenn et al., 1995; Ávila et al., 1999; Maggs \& Callow, 2003; Bulboa, 2006). Por otro lado, este sistema de cultivo necesita una mayor cantidad de tiempo (3 a 4 meses) de mantención en condiciones controladas antes de ser llevado al mar (Bulboa, 2006) pudiendo implicar además, la manipulación de equipos (microscopios, estereoscopios) para llevar a cabo ciertos procedimientos de monitoreo y observación de las etapas de cultivo (esporulación, asentamiento) lo que podría ser restrictivo para algunas personas.

El objetivo de este este estudio fue evaluar las mejores condiciones en que se produce liberación de tetrasporas de Chondracanthus chamissoi cuando es sometida a estrés durante tres horas de desecación y luego es colocada en matraces con agua de mar enriquecida para inducir la liberación de espora

\section{Materiales y métodos}

\section{Colecta y preparación de material biológico}

Se realizó la colecta de especímenes tetrasporofiticos en Playa Mendieta $\left(14^{\circ} 3,5^{\prime} \mathrm{S}\right)$ dentro de la Reserva Nacional de Paracas a profundidades de 1,5-2,5 m mediante buceo autónomo, los mismos fueron limpiados de toda impureza en campo mediante lavado mecanico con agua de mar para luego ser transportados en contenedores térmicos (cooler) en condiciones de baja temperatura $\left(5-10^{\circ} \mathrm{C}\right)$ hasta el Laboratorio de Esporocultivo de la empresa Acuicola Mares del Sur S.A.C. en el C.P. La Puntilla, Pisco durante el mes de junio del 2015. Una vez en el laboratorio, los individuos fueron lavados con agua destilada $\mathrm{y}$ posteriormente con agua de mar para evitar algún tipo de estrés osmótico, fueron secados con paños absorbentes y finalmente colocados en bandejas donde fueron sometidos a condiciones de desecación por 3 horas.

\section{Obtención del inoculo de tetrasporas}

Para inducir a la esporulación, 120 gramos de la fase tetrasporofitica fueron colocados equitativamente en 12 matraces de 1 litro con agua de mar (filtrada a 0,5 $\mu \mathrm{m}$ y autoclavada), los cuales fueron sometidos a tres experimentos de fotoperiodo (T1, T2 y T3) de 16:08, 12:12 y 08:16 (L:O) durante 24 horas (tres matraces por experimento). Evidenciado la liberación de tetrasporas, se procedió a tomar muestras de agua de los distintos experimentos y se realizó el conteo de las mismas en una cámara de Neubauer.

\section{Inoculación de tetrasporas}

Habiendo conocido la densidad de esporas, el contenido de cada matraz fue agregado a baldes con 17 litros de agua de mar esterilizada y enriquecida con nutriente comercial Bayfolan ( $(1 \mathrm{ml} / \mathrm{L})$. En cada balde se colocó bastidores cilíndricos de PVC rodeado de cuerdas de polipropileno de 3/16 pulgadas los que finalmente fueron sometidos a aireación constante desde el interior del bastidor, fotoperiodos de 16:08, 12:12 y 08:16 (L:O) y una temperatura de $21,0 \pm 1,2{ }^{\circ} \mathrm{C}$ durante 28 días. Datos sobre número de esporas asentadas por centímetro de cuerda (nEA), diámetro promedio del disco de germinación (dDG) y porcentaje promedio de discos con formación de microtalo (\%dM) fueron tomados con ayuda de un microscopio óptico aleatoriamente de cada bastidor.

\section{Análisis estadístico}

Se evaluó la normalidad y la homocedasticidad con la prueba de Shapiro-Wilks y Levene respectivamente. Los datos encontrados fueron sometidos mediante a un análisis de varianza (ANOVA) para evaluar si existen diferencias significativas entre los tratamientos. Posteriormente, se utilizó la prueba de Fisher en los experimentos a fin de encontrar diferencias significativas. Todas las pruebas se realizaron haciendo uso del paquete estadístico MINITAB 16.

\section{Resultados y discusión}

Se encontró que existen diferencias significativas $(p<0,05)$ en la cantidad de tetrasporas liberadas por cada tratamiento de fotoperiodo (T1: $0,98^{*} 10^{4} \mathrm{esp} / \mathrm{ml} ; \mathrm{T} 2: 1,65^{*} 10^{4} \mathrm{esp} / \mathrm{ml}$; T3: $\left.2,54 * 10^{4} \mathrm{esp} / \mathrm{ml}\right)$. Los valores de numero de esporas 
asentadas por centimetro de cuerda (T1: $25,3 \pm 0,3 ; \mathrm{T} 2$ : $26,0 \pm 0,3$; T3: $27,3 \pm 0,3)$ y diametro promedio del disco de germinación (T1: $750 \pm 35 \mu \mathrm{m}$; T2: $730 \pm 20 \mu \mathrm{m}$; T3: $775 \pm 20 \mu \mathrm{m})$ no presentaron diferencias significativas $(\mathrm{p}>0.05)$ en todos los sistemas trabajados, sin embargo si se presentaron diferencias significativas $(\mathrm{p}<0,05)$ en los valores porcentaje promedio de discos con formación de microtalo (T1: 79,5 $\pm 5,5 \%$ ).

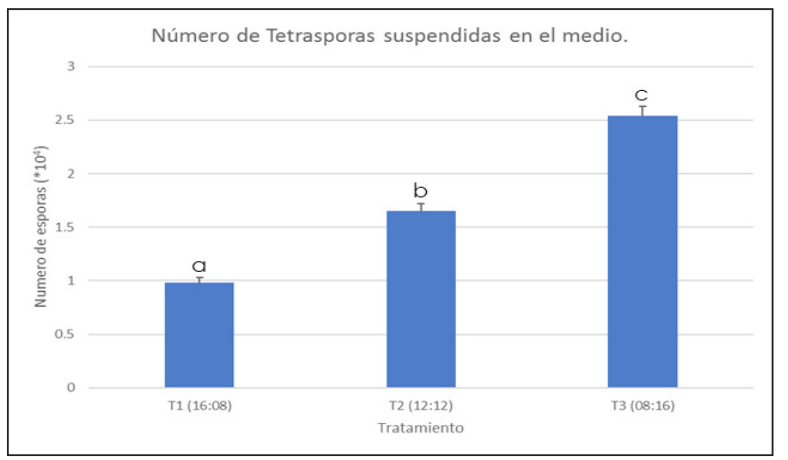

Figura 1. Número de tetrasporas suspendidas en medio acuático luego de la inducción a la esporulación según tratamiento de fotoperiodo. Letras diferentes indican diferencias significativas entre tratamientos $(p<0,05)$

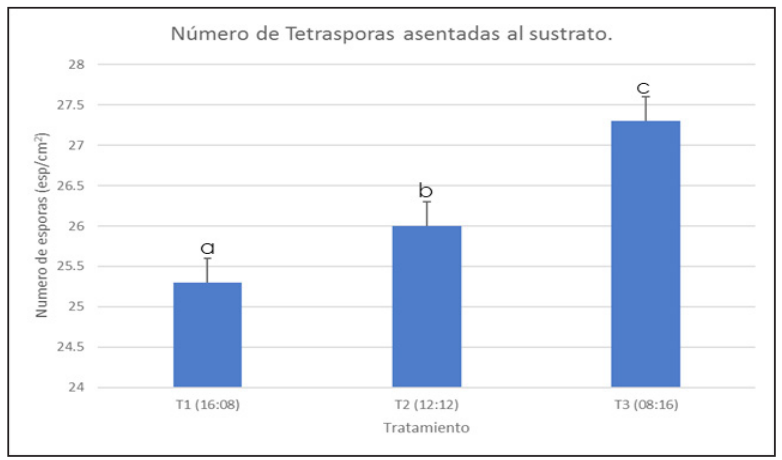

Figura 2. Número de tetrasporas asentadas sobre sustrato según tratamiento de fotoperiodo. Letras diferentes indican diferencias significativas entre tratamientos $(p<0,05)$

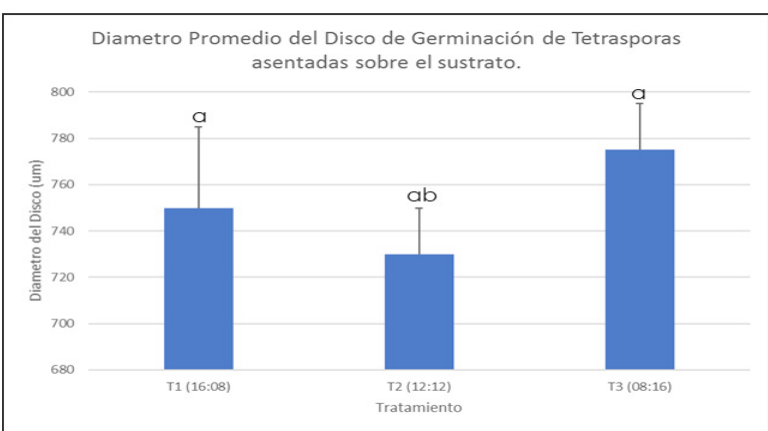

Figura 3. Diámetro promedio del Disco de Germinación formado a partir de tetrasporas asentadas según tratamiento de fotoperiodo. Letras diferentes indican diferencias significativas entre tratamientos $(p<0,05)$

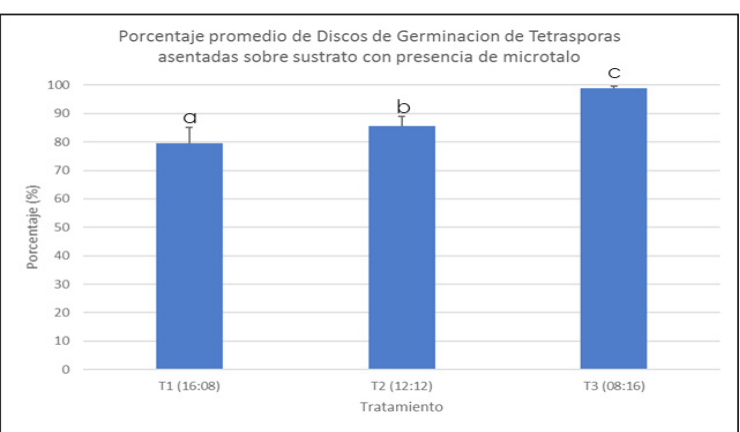

Figura 4. Porcentaje promedio de Disco de Germinación formado a partir de tetrasporas asentadas con formación de microtalo según tratamiento de fotoperiodo luego de 28 días de experimentación. Letras diferentes indican diferencias significativas entre tratamientos $(p<0,05)$

Es conocido que el comportamiento reproductivo de las macroalgas muchas veces es influenciado por las condiciones fisicoquímicas ambientales. En la zona sur del Perú el comportamiento de Chondracanthus a nivel poblacional presenta una mayor cantidad de individuos tetrasporofitos maduros a finales del verano e inicios del otoño mientras que a inicios de la primavera es conocida la mayor presencia de organismos carposporofitos maduros. Ello hace suponer que los picos de liberación de esporas tetrasporofiticas y carposporofiticas sucede en los meses de Mayo y Setiembre respectivamente. Según la época estacional la cantidad de horas luz es variable y ello repercute en el normal desarrollo de las praderas naturales de $C$. chamissoi. En nuestros resultados la menor cantidad de horas luz se vio reflejado en una mayor liberación de esporas, mejores tasas de asentamiento y posterior crecimiento de los discos de germinación asentados. Con respecto al asentamiento y crecimiento de tetrasporas en condiciones controladas de laboratorio pocos autores han realizado trabajos a fines, sin embargo las bases para el desarrollo de los mismos han sido estudiado en Perú y Chile. Acleto (1986) menciona que los aspectos biológicos de $C$. chamissoi son influenciados fuertemente por la variación de temperatura del agua y la cantidad de nutriente presente en el medio donde se desarrolla una pradera natural, pero que los picos de liberación de esporas se ubicaban en épocas de cambio brusco de incidencia solar.

Bulboa \& Macchiavello (2001) precisan además que las esporas pueden presentar características fototaxicas y que su asentamiento es influencia por la cantidad de horas luz y la temperatura del agua donde se desarrolla. Bulboa (2006) demuestra en las bases tecnológicas para el cultivo de C. chamissoi que el asentamiento de esporas además de poseer características fototaxicas también posee cualidades bioreceptoras en donde es necesario un biofilm el cual puede poseer una caracterización biológica acorde a las condiciones ambientales en donde se produzca. Finalmente, el trabajo mas reciente trabajado en asentamiento y crecimiento de tetrasporas fue realizado por Avila et al (2011) en donde asentaron y desarrollaron tetrasporas 
viables en condiciones controladas de laboratorio sobre medio agar nutrido encontrando crecimiento de microtalos con tasas de crecimiento de hasta $22 \% \cdot$ dia $^{-1}$. Los resultados encontrados son favorables y demuestra la posibilidad de realizar el cultivo de individuos gametofitos a partir de tetrasporas asentadas aunque es preciso realizar repeticiones de los experimentos en épocas primaverales para realizar comparaciones estacionales.

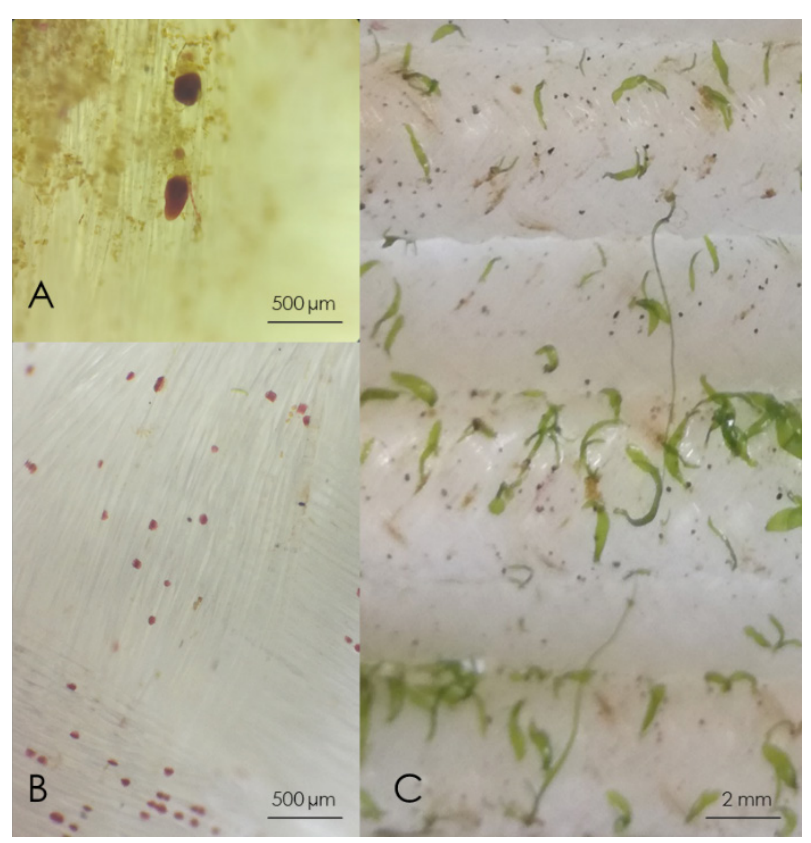

Figura 5. Asentamiento de tetrasporas sobre sustrato artificial. A. Disco de germinación con crecimiento de microtalo. B. Esporas asentadas y posterior formación de discos de germinación. C. Vista macroscópica de discos de germinación asentados sobre sustrato artificial luego de 28 días de experimentación

\section{Conclusión}

Del estudio realizado se concluye que el fotoperiodo 08:16 (L:O) tiene un mejor efecto en el asentamiento y crecimiento de tetrasporas de Chondracanthus chamissoi.

\section{Literatura citada}

Acleto, C. 1986. Algunos aspectos biológicos de Gigartina chamissoi (C. Ag.) J. Agardh (Rhodophyta, Gigartinales). Revista de Ciencias Universidad Nacional Mayor de San Marcos, 74(1): 38-47.

Acleto, C. 1971. Algas marinas del Perú de importancia económica. Serie de divulgación Nro. 5. Universidad Nacional Mayor de San Marcos. Lima - Perú. Pág.: 13

Acleto, C. y Zúñiga. 1998. Introducción a las algas. Universidad Nacional Mayor de San Marcos. 1 Ed. Lima, Perú. 263p.

Agrawal, S. C. 2009. Factors affecting spore germination in Algae - review. Folia Microbiologic, 54(4): 273-302.

Alveal, K.; Romo, H. y Werlinger, C. 1995. Cultivo de
Gracilaria a partir de esporas. En Alveal, K.; Ferrario, M. E.; Oliveira, E. C.; Sar, E. 1995. Manual de Métodos ficológicos. 599-609p. Universidad de Concepción. Chile.

Alveal, K.; Romo, H.; Werlinger, C.; Vallejos, P.; Alveal, K. y Alveal, A. 1999. Desarrollo inicial de Chondracanthus chamissoi sobre sustrato artificial. Resúmenes del Congreso Latinoamericano de Ciencias del Mar. Perú. 14-16p.

Alveal, K. 2001. Estrategias reproductivas de Rhodophyta y sus nexos con biodiversidad. En: Alveal, K; Antezana, T. Sustentabilidad de la biodiversidad. Universidad de Concepción-Chile. 367-388p.

Ávila, M.; Ask, E.; Rudolph, B.; Nuñez, M. and Norambuena, R. 1999. Economic feasibility of Sarcothalia (Gigartinales, Rhodophyta) cultivation.

Ávila, M.; Piel, M.; Cáceres, J. and Alveal, K. 2011. Cultivation of the red alga Chondracanthus chamissoi: sexual reproduction and seeding production in culture under controlled conditions. J. Appl. Phycol. 23:529536.

Barrientos, E. y Otaíza, D.R. 2014. Juveniles generados a partir de esporas no asentadas de Chondracanthus chamissoi (Rhodophyta, Gigartinales) presentan capacidad de adhesión al sustrato. Nota científica. Revista de Biología Marina y Oceanografía, 49(1): 135-140.

Barsanti, L. and Gualtieri, P. 2014. Algae, anatomy, biochemistry and biotechnology. 2nd edition. Istituto di Biofisica. Pisa - Italy.

Bulboa, R. C. and Macchiavello, E. J. 2001. The effects of the light and temperature on different phases of the life cycle in the carrageenan producing alga Chondracanthus chamissoi (Rhodophyta, Gigartinales). Botanica Marina, 44: 371-374.

Bulboa, C., Macchiavello, J., Oliveira, E. and Fonck, E. 2005. First attempt to cultivate the carrageenanproducing seaweed Chondracanthus chamissoi (C. Agardh) Kützing (Rhodophyta; Gigartinales) in Northern Chile. Aquac. Res. 36: 1069-1074.

Bulboa, C. and Macchiavello, J. 2006. Cultivation of cystocarpic, tetrasporic and vegetative fronds of Chondracanthus chamissoi (Rhodophyta, Gigartinales) on ropes at two localities in Northern Chile. Invest. Mar., Valparaíso, 34(1): 109-112.

Bulboa, C.C. 2006. Bases bio-tecnológicas para o cultivo de Chondracanthus chamissoi, uma alga vermelha de importância econômica da costa chilena. Tese apresentada ao Instituto de Biociências da Universidade de São Paulo, para a obtenção de Título de Doutor em Ciências, na Área de Botânica. Brasil.

Bulboa, C.; Macchiavello, J.; Oliveira, E. and Véliz, K. 2008. Grow rate differences between four Chilean populations of edible seaweed Chondracanthus chamissoi (Rhodophyta, Gigartinales). Aquaculture Research, 39: 1550-1555. 
Bulboa, C.; Macchiavello, J.; Véliz, K. and Oliveira, C. E. 2010. Germination rate and sporeling development of Chondracanthus chamissoi (Rhodophyta, Gigartinales) varies along a latitudinal gradient on the coast of Chile. Aquatic Botany, 92: 137-141.

Bulboa, C.; Véliz, K.; Sáez, F.; Sepúlveda, C.; Vega, L. and Macchiavello, J. 2013. A new method for cultivation of the carragenophyte and edible red seaweed Chondracanthus chamissoi based on secondary attachment disc: Development in outdoor tanks. Aquaculture, 410-411: 86-94.

Buschmann, A. H.; Kuschel, A. F.; Vergara, A. P.; Schulz, J. 1992. Intertidal Gracilaria farming in southern Chile: differences of the algal provenience. Aquatic Botany, 42: 327-337.

Buschmann, H. A; Vásquez, J. A; Osorio, P.; Reyes, E.; Filún, L.; Hernández-Gonzáles, M.C. and Vega, A. 2004. The effect of water movement, temperature and salinity on abundance and reproductive patterns of Macrocystis spp. (Phaeophyta) at differents latitudes in Chile. Marine Biology, 145: 849-862

Calderón, M.; Rámirez, M.H. y Bustamante, D. 2010. Notas sobre tres especies de Gigartinaceae (Rhodophyta) del litoral peruano. Rev. peru. biol. 17(1): 115- 121.

Carbajal, W.; Galán J. y De la Cruz, J. 2005. Prospección del recurso Chondracanthus chamissoi "cochayuyo" en la playa de Huanchaco (Trujillo), Junio - Julio 2005. Instituto del Mar del Perú.

Carbajal, W.; De la Cruz, J. y Galán, J. 2005. Prospección del recurso Chondracanthus chamissoi "cochayuyo" en la Caleta de Chérrepe (Lambayeque), Julio 2005. Instituto del Mar del Perú.

Chopin, T.; Pringle, J. D. and Semple, R. E. 1988 Reproductive capacity of dragraked and non dragraked Irish moss (Chondrus crispus Stackhouse) beds in the southern Gulf of St. Lawrence. Can. J. Fish. Aquat. Sci. 49: 758-766.

Dawson, Y; Acleto, C. and Foldvik, N. 1964. The seaweeds of Peru. Nova Helwigia. 13: 1-111.

Edding, M. E. Cultivo de Gracilaria en estanques. 1995 En Alveal, K.; Ferrario, M. E.; Oliveira, E. C.; Sar, E. 1995. Manual de Métodos Ficológicos. Universidad de Concepción. Chile. 577-597p.

Egan, S.; Thomas, T.; Holmström, C. and Kjelleberg, S. 2000. Phylogenetic relationship and antifouling activity of bacterial epiphytes from the marine alga Ulva lactuca. Environ. Microbiol. 2:343-347.

Fonck, E.; Martínez R.; Vásquez, J. and Bulboa, C. 2007. Factors that affect the re-attachment of Chondracanthus chamissoi (Rhodophyta, Gigartinales) thalli. J Appl Phycol.

Garza-Sánchez, F.; Zertuche-Gonzáles, J.A. and Chapman, D. J. 2000. Effect of temperature and irradiance on the release, attachment and survival of spores of Gracilaria pacifica Abbott (Rhodophyta). Botanica Marina, 43:
205-212.

Glenn, E. P.; Moore, D.; Fitzsimmons, K. and Azevedo, C. 1996. Spore culture of the edible red seaweed, Gracilaria parvispora (Rhodophyta). Aquaculture, 142: $59-74$

Gonzales, J. and Meneses, I. 1996. Differences in the early stages of development of gametophytes and tetrasporophytes of Chondracanthus chamissoi (C.Ag.) Kützing from Puerto Aldea, northern Chile.

Hanisak, M.D., Littler, M.M. and Littler, D.S. 1990. Application of the functional form model to the culture of seaweeds. Hydrobiologia: 204/205: 73-77.

Hayashi, L.; Bulboa, C.; Kradolfer, P.; Soriano G. and Robledo D. 2013. Cultivation of red seaweeds: a Latin American perspective. J. Appl. Phycol. 26:719-729.

Israel, A. 1995. Determinación de la producción primaria en macroalgas marinas. En Alveal, K.; Ferrario, M. E.; Oliveira, E. C.; Sar, E. (1995). Manual de Métodos Ficológicos. 1995. 397-416p. Universidad de Concepción. Chile.

Lobban, R. and Harrison, P. 1994. Seaweed Ecology and Physiology. Cambridge University Press.

Macaya, E. 2001. Uso de carpoesporas para el cultivo de Chondracanthus chamissoi (C. Agardh) Kützing 1843 (Rhodophyta; Gigartinales): Inducción a la esporulación y fijación sobre diferentes sustratos. Tesis Doctoral. Universidad Católica del Norte.

Macchiavello, J.; Bulboa, C. and Edding, M., 2003. Vegetative propagation and spore recruitment in the carrageenophyte Chondracanthus chamissoi (Rhodophyta, Gigartinales) in northern Chile. Phycological Research, 51: 45-50.

Maggs, A. A. and Callow, M. E. 2003. Algal Spores. Encyclopedia of life sciences. Nature Publishing group, London, version 1.0, 1-6p.

Oliveira, E. C.; Paula, E. J.; Plastino, E. M. y Petti, R. 1995. Metodologías para el cultivo no axénico de macroalgas marinas in vitro. En Alveal, K.; Ferrario, M. E.; Oliveira, E. C.; Sar, E. 1995. Manual de Métodos Ficológicos. 429-447p. Universidad de Concepción. Chile.

Otaíza, D. R. and Fonseca, G. F. 2011. Effect of dissolved calcium on the formation of secondary attachment structures in different types of branches of Chondracanthus chamissoi (Rhodophyta, Gigartinales). Nota de investigación. Revista de Biología Marina y Oceanografía.,46 (2): 263-268.

Pacheco-Ruíz, I.; Zertuche-González, J. A.; ArroyoOrtega, E.; Valenzuela-Espinoza, E. 2004. Agricultural fertilizers as alternative culture media for biomass production of Chondracanthus squarrulosus (Rhodophyta, Gigartinales) under semi-controlled conditions. Aquaculture, 240: 201-209.

Pacheco-Ruíz, I.; Zertuche-González, J. A. and EspinozaÁvalos, J. 2005. The role of the secondary attachment 
discs in the survival of Chondracanthus squarrulosus (Gigartinales, Rhodophyta). Phycologia, 44: 629-631.

Pariona, E. 2004. Determinación de la abundancia de las fases reproductivas de Chondracanthus chamissoi (C. Agardh) Kutzing y reclutamiento sobre sustrato calcáreo en una pradera de playa Mendieta. Reserva Nacional de Paracas - Perú. Tesis para optar el título de biólogo. Universidad Nacional Agraria La Molina, Lima, Perú.

Ramírez, M.E. y Santelices, B. 1991. Catálogo de las algas marinas bentónicas de la costa temperada del Pacífico Sudamericano. Monografías Biológicas. Facultad de Ciencias Biológicas, Pontificia Universidad Católica de Chile. Publicaciones Periódicas Vicerrectoría Académica, Santiago de Chile, 5: 6-19.

Redmond, S.; Green, L.; Yarish, C. H.; Kim, J. and Neefus, C. 2014. New England, Seaweed culture handbook. Nursery systems. University of Connecticut \& University of New Hampshire.

Riofrío, L. 2003. Efecto de la variabilidad térmica sobre la biología vegetativa y reproductiva de Chondracanthus chamissoi (Agardh) Kützing (Rhodophyta) en la bahía de Ancón, Perú. Tesis para optar el título de Biólogo. UNMSM, Lima, Perú. 41 p.

Sáez, F.; Macchiavello, J; Fonck, E. \& Bulboa, C. 2008. The role of the secondary attachment disc in the vegetative propagation of Chondracanthus chamissoi (Gigartinales; Rhodophyta). Aquatic Botany, 89: 63-65.

Santelices, B. 1990. Patterns of reproduction, dispersal and recruitment in seaweeds. Oceanography Marine Biology Annual Review, 28: 177-276.

Santelices, B. and Varela, D. 1993. Intra-clonal variation in the red seaweed Gracilaria chilensis. Marine Biology, 116: 543-552.

Salinas, J. M. and Valdés, L. 1993. Influence of temperature and photoperiod on the re-attachment process of Gelidium sesquipedale (Clem.) Born. Et Thur. (Gelidiales: Rhodophyta). Journal of Applied Phycology, 317-326.

Silva-aciares, F.; Riquelme, C. 2008. Inhibition of attachment of some fouling diatoms and settlement of Ulva lactuca zoospores by film forming bacterium and their extracellular products isolated from biofouled substrata in Northern Chile. Electronic J.Biotechnol. 11: $1-11$.

Universidad Católica del Norte 2012. Manual de Cultivo de Chondracanthus chamissoi "Chicorea de Mar". Fondef. Chile.

Vásquez, J. A.; Vega, J. M. 2001. Chondracanthus chamissoi (Rhodophyta, Gigartinales) in northern Chile: ecological aspects for management of wild populations. Journal of Applied Phycology, 13: 267-277.

Vega, J.M.A.; Meneses, I. 2001. Seasonal and spatial monitoring of productivity and of reproduction of Chondrus canaliculatus (Gigartinales, Rhodophyta) from Chile. Botanica Marina, 44: 571-581.

Yang, M.Y.; Macaya, E.C. y Kim, M.S. 2015. Molecular evidence forverifying the distribution ofChondracanthus chamissoi and C. teedei (Gigartinaceae, Rhodophyta). Botanica Marina, 58(2): 103-113. 